\title{
AlphaLogD determination: a new generation Reversed-Phase HPLC method to measure lipophilicity on neutral and basic small and Beyond-Rule-of-Five compounds.
}

Daniel Katz ${ }^{(a)}$, Kate Fike ${ }^{(a)}$, Justin Longenberger ${ }^{(a)}$, Steve Placko( ${ }^{(a)}$, Laurence Philippe-Venec ${ }^{(b)}$, Andrew Chervenak $^{(a)}$

(a) Analiza Inc, 3615 Superior Avenue E, Suite 4407B, Cleveland, $\mathrm{OH}, 44114-4139$

(b) PIC Analytics, P.O. Box 192, Dexter, MI, 48130-1250

\begin{abstract}
:
Lipophilicity can be measured with different methods, such as Shake-Flask or liquid chromatography. HPLC presents the advantage of overcoming solubility issues and therefore extending the range of lipophilicity to high values. A specific HPLC method, called ELOgD, had been developed 20 years ago on a $\mathrm{C}_{16}$-amide stationary phase, enhancing hydrophobic and hydrogen bond interactions to mimic octanolwater partition. The emergence of novel stationary phases added to the need for a less complex mobile phase has led to the development of a new HPLC assay called alphaLogD, applicable to neutral and basic compounds at $\mathrm{pH}$ 7.4, that combines superficially porous particles, a high number of equilibriums between solutes and stationary phase, and results in a lower number of isocratic methods to determine the logk' ${ }_{w}$ at a higher throughput. Statistical studies have been run to successfully evaluate the alphaLogD method compared to the Shake-Flask method and to allow this lipophilicity measurement into the socalled Beyond-Rule-of-5-molecules space.
\end{abstract}

Keywords: High Performance Liquid Chromatography, Superficially Porous Particle, Shake-Flask method, Lipophilicity, Beyond-Rule-of-5

\section{Introduction}

Lead Discovery is an iteration of optimizations of different parameters, mainly by improving potency through chemical structure modifications. These modifications are aimed to modulate in vitro physicochemical properties with the goal of optimizing in vivo oral bioavailability. Lipophilicity is one of the first physicochemical properties integrated in medicinal chemistry design, as it impacts passive permeability, metabolism, excretion, oral absorption and toxicity [1-8].

In addition, physical parameters such as solubility, the flexibility of a molecule based on the presence of rotatable bonds and on the ratio of $\mathrm{sp}^{3}$ carbons, the presence of polar groups, and the presence of Intramolecular Hydrogen Bonding are related to lipophilicity [9-11]. Finally, lipophilicity is a powerful parameter used to modulate potency via the LipE concept, allowing the study of hydrophobic effect of a structural change on both lipophilicity and potency $[12,13]$.

The importance of lipophilicity on drug design emphasizes the need for accurate determination of this property. There are multiple in silico tools that are commercially available and customizable for the determination of lipophilicity. These computational methods are often inaccurate, as logP or logD values may vary as much as two to three units when compared amongst the different software packages.

Different analytical techniques, such as solvent/water partitioning by shake-flask, partitioning in micelles by capillary electrophoresis, and liquid chromatography have been developed and miniaturized to adapt to the throughput and low amounts of available compounds at the early discovery stage $[14,15]$. 
Shake-flask is an accurate quantitative method that evaluates the amount of compound in each phase and stands as the "gold standard" in lipophilicity measurements providing lipophilicity values up to 3. However, the shake-flask technique still shows limitations for compounds of high lipophilicity, as most of the compounds will reside in the upper organic phase with limited quantification in the lower aqueous phase. In addition, low solubility of highly lipophilic compounds can generate significant variability in compounds quantification and in the final lipophilicity value. These limitations present a need for more accurate determinations of lipophilicity values, especially for very hydrophobic compounds.

Liquid chromatography on the other hand is a qualitative method, highlighting hydrophobic interactions with the lipophilic stationary phase relative to a non-retained entity. As it is less sensitive to solubility, reversed phase HPLC offers an extended range of lipophilicity values based on retention times mainly related to compound interactions and conformations in a specific environment [16]. Several conditions have been developed on different lipophilic supports to try to cover a wider range of lipophilicity with one unique method but with some limitations on the class of studied compounds [17-19]. The ELogD method, amenable to neutral and basic compounds at $\mathrm{pH} 7.4$, involves a $\mathrm{C}_{16}$ lipophilic support with embedded amide functions for higher efficiency with regard to hydrophobic interactions [20]. This reliable and reproducible assay has been developed with a complex mobile phase that contains decylamine, a masking agent to reduce secondary interactions of the solute with the support, morpholino propanesulfonic acid (MOPS) an ion-pairing agent to ensure the retention of positively charged entities, and octanol to enhance the energy of interactions present in the octanol/water system. This mobile phase has proven to be detrimental to the HPLC instrument, with the crystallization of the decylamine over time, and the limited shelf-life of the stationary phase with the saturation of the sites of the $\mathrm{C}_{16}$ amide support coated with MOPS. The need for reproducibility and reliability has led to the selection of a new generation of stationary phases, such as Superficially Porous Particle (or SPP) that contain a solid, nonporous silica core covered by a porous shell layer. SPP enhances the number of equilibriums between the stationary and the mobile phases, leading to reduced resistance to mass transfer, minimal compound diffusion, and higher column efficiency [21]. As a result, SPP allows the use of smaller particles and higher flow-rates without generating stronger back pressure. This optimized SPP technology combined to $\mathrm{C}_{16}$ lipophilic chains and an embedded amide function has led to the development of the Ascentis ${ }^{\circledR}$ Express column to generate lipophilicity data of quality similar to ELogD with significant reproducibility and a less complex mobile phase. Finally, the developed conditions on the Ascentis ${ }^{\circledR}$ Express SPP stationary phase allow for the measurement of high lipophilicity $(\log P \geq 5)$ and open new opportunities to better support the chemical space expansion towards highly lipophilic compounds, so-called Beyond-Rule-of-5 molecules.

\section{Material and Methods}

\subsection{Material for ELogD method [20]}

The ELogD HPLC method uses the Supelcosil LC-ABZ (RP-amide) column (Supelco), $5 \mu \mathrm{m}$ particle size, 50 $\mathrm{mm} \times 4.6 \mathrm{~mm}$.

The mobile phase contains decylamine $\mathrm{CH}_{3}\left(\mathrm{CH}_{2}\right)_{9} \mathrm{NH}_{2}$ (CAS 2016-57-1, from TCl, purity > 98\%), Morpholinopropane Sulfonic Acid $\mathrm{C}_{7} \mathrm{H}_{15} \mathrm{NO}_{4} \mathrm{~S}$ (CAS 1132-62-1, from J.T. Baker, purity $\geq 99.5 \%$ ), Sodium Hydroxide (Purity $>99 \%$ ) , 1-Octanol $\mathrm{CH}_{3}\left(\mathrm{CH}_{2}\right)_{7} \mathrm{OH}$ (Purity $\geq 99 \%$ Fisher), Optima HPLC grade water (Fisher), Optima HPLC grade Methyl alcohol (Fisher).

The aqueous phase is prepared by adding $0.05 \% \mathrm{v} / \mathrm{v}$ of octanol to water, $0.15 \% \mathrm{v} / \mathrm{v} \mathrm{N}$ decylamine, 20 $\mathrm{mM}$ of MOPS, and the $\mathrm{pH}$ is adjusted at 7.4 with the ammonium hydroxide. 
The organic phase contains $0.25 \% \mathrm{v} / \mathrm{v}$ of octanol in methyl alcohol.

\subsection{Material for alphaLogD method}

The alphaLogD HPLC method uses the SPP RP-Amide Ascentis $®$ Express (Supelco), $2.7 \mu \mathrm{m}$ particle size, $50 \mathrm{~mm} \times 4.6 \mathrm{~mm}$.

The mobile phase contains ammonium acetate $\mathrm{CH}_{3} \mathrm{CO}_{2} \mathrm{NH}_{4} \mathrm{HPLC}$ grade (EMD Millipore), 1-Octanol $\mathrm{CH}_{3}\left(\mathrm{CH}_{2}\right)_{7} \mathrm{OH}$ (Purity $\geq 99 \%$ Fisher), Optima HPLC grade water (Fisher), Optima HPLC grade Methyl alcohol (Fisher).

The aqueous phase is prepared by adding $0.05 \% \mathrm{v} / \mathrm{v}$ of octanol to water, and ammonium acetate at a concentration of $50 \mathrm{mM}$.

The organic phase contains $0.25 \% \mathrm{v} / \mathrm{v}$ of octanol in methyl alcohol.

\subsection{Sample preparation}

All standards used to build the calibration curves are from Sigma-Aldrich with purity $\geq 98 \%$ and are described in table 2. The standards are dissolved in DMSO (USP, Spectrum) at a concentration of $10 \mathrm{mM}$ and are diluted down to $1 \mathrm{mM}$ either with DMSO or a mixture of water/methanol 50/50 v/v.

\subsection{Instrumentation and software}

The HPLC instrument is an Agilent 1100 piloted by Chemstation Software (Version C.01.06) equipped with a quaternary HPLC pump (Model G1311A) with a micro vacuum degasser (model G1322A), a microwell plate autosampler WPALS (Model G1367A) with an injection loop of $20 \mu \mathrm{L}$, a Column thermostatic column compartment (Model G1330B), and a UV Diode Array Detector (Model G1315B).

Each isocratic method is built with the pumping system programmed to deliver constant volumes of each aqueous and organic solvent, and is delivered at $2 \mathrm{~mL} / \mathrm{min}$.

The temperatures of column compartment and autosampler are both maintained at $23 \circ \mathrm{C}$.

Statistical Analyses: Linear regressions, ANOVAs, parallel lines analysis, and Bland-Altman plots were generated using SigmaPlot version 14.5, from Systat Software, Inc., San Jose California USA, (www.systatsoftware.com).

\section{Theory and Calculations}

Lipophilicity models by Reversed-Phase Liquid Chromatography have been proven to be indirectly related to the Shake-Flask model where the compound partition between octanol and water logKow is driven by an ensemble of diverse types of interactions, as described by the Linear Solvation Energy Relationship, LSER established by Abraham [22] defined by Eq. (1):

$\log _{\mathrm{ow}}=c+e E+s S+a A+b B+w$

Each specific intermolecular interaction is represented by the product of solute descriptor with the complementary system constant related to the solute. These solute descriptors respectively highlight the excess molar refraction $E$, the polarizability $S$, the effective hydrogen-bond acidity $A$, the effective hydrogen-bond basicity $B$, and the McGowan's characteristic volume $V$. The constants stand for the 
system contributions related to the solute, such as $e$ for the capacity of the system to interact with the electron lone pair interactions, $s$ for the ability to form dipole-dipole interactions with the solute, $a$ and $b$ for the capacity of forming hydrogen bonds, $v$ for the ability of the solute to create cavities through cohesion and dispersion interactions in each phase, and $c$ being a system constant. Parallel to the ShakeFlask partition, the LSER model can be applied to a reverse-phase liquid chromatographic system with each intermolecular interaction contributing to the retention of the solute. In both cases, each system constant is calculated with multiple linear regression analyses for a selected group of solutes with known descriptors. The resulting logk' is the qualitative and quantitative description of the intermolecular interactions in the partition process between octanol and water or in the equilibrium of the solute between the mobile phase and the stationary phase in a liquid chromatographic system [23].

A comparative study of LSER system constants calculated from the octanol-water partition and from two chromatographic systems involving the Supelcosil LC-ABZ and the Ascentis stationary phases, respectively, highlights the similarities of the interactions of the two chromatographic processes with the octanol-water system in the lipophilicity determination [24-26] (Table 1, Rows (a) and (b)). The magnitude of each system constant is related to the importance of the interaction in the partition or retention process, and the positive or negative sign is indicative of the interaction with either the stationary phase or the mobile phase in the chromatographic system. The interactions study on the RP amide support emphasizes the positive contribution of the Hydrogen Bond Acidity (or Hydrogen Bond donor) of the solute with the stationary phase compared to the $C_{18}$ support (Table 1, Rows (c) and (d)). The compared ratios of the system constant between the RP-amide chromatographic system and the Octanol-Water partition are nearly identical and therefore a correlation model can be built between partition and retention, defined by Eq. (2):

$\log \mathrm{K}_{\text {ow }}$ or $\log \mathrm{P}=\mathrm{p}+\mathrm{qlogk}^{\prime}$

$\log \mathrm{K}_{\mathrm{ow}}=$ partition coefficient between octanol and water = Lipophilicity.

logk' = solute retention between stationary phase and mobile phase in a reversed-phase liquid chromatography system.

$p$ and $q=$ linear regression coefficients

Table 1

Comparison of system constants of LC-ABZ and Ascentis ${ }^{\circledR}$ Express stationary phases with system constants of octanol-water partition.

\begin{tabular}{|c|c|c|c|c|c|}
\hline Separation system & Sepa & tion $\mathrm{cc}$ & & & \\
\hline & $v$ & $e / v$ & $s / v$ & $a / v$ & $b / v$ \\
\hline Octanol-water [24] & 3.81 & 0.15 & -0.28 & 0.01 & -0.9 \\
\hline $\begin{array}{l}\text { Supelcosil } \\
\text { LC-ABZ }^{(a)}[24]\end{array}$ & 3.48 & 0.12 & -0.27 & -0.01 & -0.89 \\
\hline $\begin{array}{l}\text { Ascentis } \\
\text { RP-Amide }^{(b)} \text { [25] }\end{array}$ & 4.15 & 0.09 & -0.23 & -0.03 & -0.84 \\
\hline $\begin{array}{l}\text { Ascentis } \\
\text { RP-Amide }^{(c)}[26]\end{array}$ & 2.23 & 0.07 & -0.17 & 0.03 & -1.12 \\
\hline Ascentis $C_{18}{ }^{(\mathrm{d})}[26]$ & 2.30 & 0.12 & -0.32 & -0.11 & -0.91 \\
\hline
\end{tabular}

(a) Supelcosil LC-ABZ system:

- $\quad$ Embedded RP -Amide stationary phase, coated with octanol, 
- Mobile phase: $20 \mathrm{mM}$ MOPS pH 7.4 saturated with octanol $-15 \%$ to $70 \%$ of methanol containing $0.25 \% \mathrm{v} / \mathrm{v}$ octanol.

(b) Ascentis RP-amide:

- Embedded RP-Amide stationary phase,

- Mobile Phase:20 mM Sodium Phosphate buffer $\mathrm{pH} 7$ saturated with octanol- isocratic methods from 40 to $55 \%$ of Methanol.

(c) Ascentis RP-amide:

- Embedded RP-Amide stationary phase,

- Mobile Phase:20 mM Sodium Phosphate buffer $\mathrm{pH} 2-$ isocratic method 75/25 \% v/v of acetonitrile.

(d) Ascentis $\mathrm{C}_{18}$ :

- Mobile Phase:20 mM Sodium Phosphate buffer $\mathrm{pH}$ 2- isocratic method 75/25 \% v/v of acetonitrile.

The solute retention logk' on the stationary phase is directly related to its interactions between the stationary phase and the mobile phase and is expressed as the capacity factor, Eq. (3):

$\log k^{\prime}=\log \left[\left(t_{R}-t_{0}\right) / t_{0}\right]$

$t_{R}=$ elution time of the solute on the packed stationary phase, at a given composition of the mobile phase.

$t_{0}=$ elution of a non-retained compound on the packed stationary phase independent of the composition of the mobile phase.

A change in the mobile phase composition will induce a change in the retention time, and we can apply the Snyder Linear Solvent Strength model (LSS) to assume a direct linear relationship between the solute retention and a binary mobile phase composition, as shown in Eq. (4):

$\log k^{\prime}=\log k^{\prime}{ }_{w}-S \Phi$

$\log \mathrm{k}^{\prime}{ }_{\mathrm{w}}=$ extrapolated value of logk' at $100 \%$ of water.

$\mathrm{S}=$ Solute dependent solvent strength parameter.

$\Phi=$ ratio of organic modifier in the mobile phase of the chromatographic system.

Applying the theory regarding retention of a solute in a chromatographic system and based on our previous knowledge of chromatographic lipophilicity determination on LC-ABZ stationary phase, we are developing a new methodology on the embedded $\mathrm{C}_{16}$-amide column RP-Ascentis $\AA$ Express with Superficially Porous Particle to generate alphaLogD on neutral and basic compounds.

\section{Results and Discussion}

\subsection{Linear Solvent Strength model}

The LSS concept has been validated through the interactions of tetracaine of known lipophilicity of 2.29 and eluted on the RP-Ascentis $®$ Express column with isocratic mobile phases containing $50 \mathrm{mM}$ Ammonium Acetate at $\mathrm{pH} 7.4$ and $0.25 \% \mathrm{v} / \mathrm{v}$ octanol for the aqueous phase and $0.25 \% \mathrm{v} / \mathrm{v}$ octanol in methanol for the organic phase. Seven isocratic methods containing respectively $20 \%, 30 \%, 40 \%, 45 \%$, $60 \%, 65 \%$, and $75 \%$ of organic content have been screened and the logk' of tetracaine is reported as a linear function of the organic solvent strength (Figure 1), confirming the use of equations (2) and (4) for the respective determination of $\operatorname{logk}^{\prime}{ }_{w}$ and the final $\log D$ for charged entities or LogP for neutral ones. 


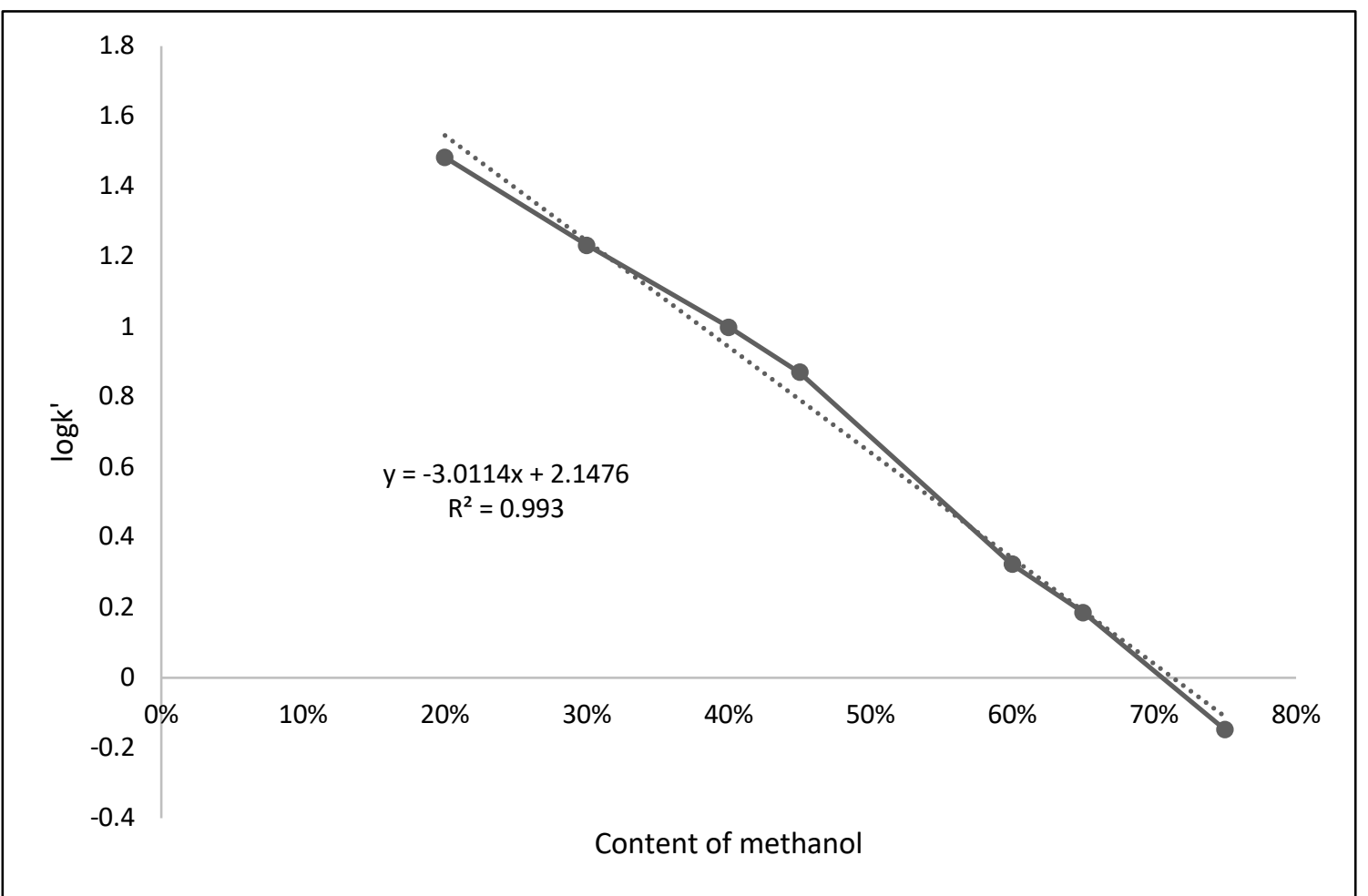

Fig. 1. Correlation of retention time of tetracaine with content of methyl alcohol in the mobile phase.

With a pKa measured at 8.78 , the basic tetracaine is partially ionized in the mobile phase at $\mathrm{pH} 7.4$ and, in addition to the hydrophobic interactions with the lipophilic chains of the stationary phase, the presence of the hydrogen donor contributes to the retention of the compound based on its interactions with the carbonyl group of the amide function of the stationary phase [26].

\subsection{Choice of standards}

The main goal of the study is to create a linear model between the distribution in an interaction-based system, such as Reversed Phase Liquid Chromatography, and the partition between two non-miscible liquid phases, such as octanol-water, for compounds of known diverse lipophilicities. The choice of the standards is based on the potential combination of least one hydrogen donor at the studied $\mathrm{pH}$ and of lipophilic chains to create interactions with the RP-Amide stationary phase that will result in different retention times. The selected standards, mainly basic, have an extended range of measured pKa leading to the presence of neutral and ionized forms in the mobile phase of $\mathrm{pH} 7.4$ (Table 2). 20 standards on a lipophilicity range from -1 to 6 , described in the literature, are selected (Figure 2 ) and studied in the RPAscentis $\circledR^{\circledR}$ Express system. 
Table 2

Standards used for the calibration curve of alphaLogD.

\begin{tabular}{|c|c|c|c|c|c|c|c|}
\hline Compound & CAS no & MW & $\begin{array}{l}\text { \# H } \\
\text { donor }\end{array}$ & $\begin{array}{l}\text { pKa }{ }^{*} \\
\text { measured }\end{array}$ & $\begin{array}{l}\text { Literature } \\
\log \mathrm{D}[20]\end{array}$ & $\begin{array}{l}\text { ELogD } \\
{[20]}\end{array}$ & AlphaLogD \\
\hline Procainamide & $51-06-9$ & 235.3 & 2 & $\begin{array}{l}2.73 \\
9.42\end{array}$ & -0.91 & -0.72 & -0.98 \\
\hline Allopurinol & $315-30-0$ & 136.1 & 2 & 9.20 & -0.44 & -0.06 & -0.86 \\
\hline Acebutolol & $37517-30-9$ & 333.4 & 3 & 9.70 & -0.29 & -0.53 & 0.27 \\
\hline Metrodinazole & $443-48-1$ & 171.5 & 1 & 2.49 & -0.02 & 0.08 & -0.31 \\
\hline Antipyrine & $60-80-0$ & 188.2 & 0 & $<1.7$ & 0.38 & 0.29 & 0.02 \\
\hline Acetaminophen & $103-90-2$ & 151.2 & 2 & 9.38 & 0.51 & 0.31 & 0.10 \\
\hline Alprenolol & $13655-52-2$ & 249.3 & 2 & 9.72 & 0.97 & 0.59 & 1.53 \\
\hline Triamterene & $396-01-0$ & 253.3 & 3 & 6.39 & 1.21 & 1.14 & 1.01 \\
\hline Hydrocortisone & $50-23-7$ & 362.5 & 3 & $>12$ & 1.55 & 1.57 & 1.29 \\
\hline \multirow[t]{2}{*}{ Quinidine } & $56-54-2$ & 324.4 & 1 & 4.36 & 2.04 & 1.61 & 1.93 \\
\hline & & & & 8.69 & & & \\
\hline \multirow[t]{2}{*}{ Tetracaine } & $94-24-6$ & 264.4 & 1 & 1.93 & 2.29 & 2.49 & 2.75 \\
\hline & & & & 8.78 & & & \\
\hline \multirow[t]{2}{*}{ Omeprazole } & $73590-58-6$ & 345.4 & 1 & 6.34 & 2.30 & 2.03 & 2.22 \\
\hline & & & & 9.07 & & & \\
\hline Imipramine & $50-49-7$ & 280.4 & 1 & 9.68 & 2.40 & 2.53 & 2.73 \\
\hline \multirow[t]{2}{*}{ Clozapine } & $5786-21-0$ & 326.8 & 1 & 4.10 & 3.13 & 3.60 & 3.48 \\
\hline & & & & 7.94 & & & \\
\hline Triflupromazine & $146-54-3$ & 352.4 & 1 & 9.39 & 3.61 & 3.69 & 3.85 \\
\hline Bifonazole & $60628-96-8$ & 310.4 & 0 & 6.28 & 4.77 & 5.24 & 4.90 \\
\hline Diethylstilbesterol & $56-53-1$ & 268.3 & 2 & 9.77 & 5.07 & 4.95 & 4.83 \\
\hline Clotrimazole & 23593-75-1 & 344.8 & 0 & 5.99 & 5.20 & 4.91 & 4.67 \\
\hline Tolnaftate & $2398-96-1$ & 307.4 & 0 & $<1.2$ & 5.40 & 5.46 & 5.25 \\
\hline Amiodarone & $1951-25-3$ & 645.3 & 1 & 7.85 & 6.10 & 6.33 & 6.42 \\
\hline
\end{tabular}

*pKa measured by Capillary Electrophoresis 


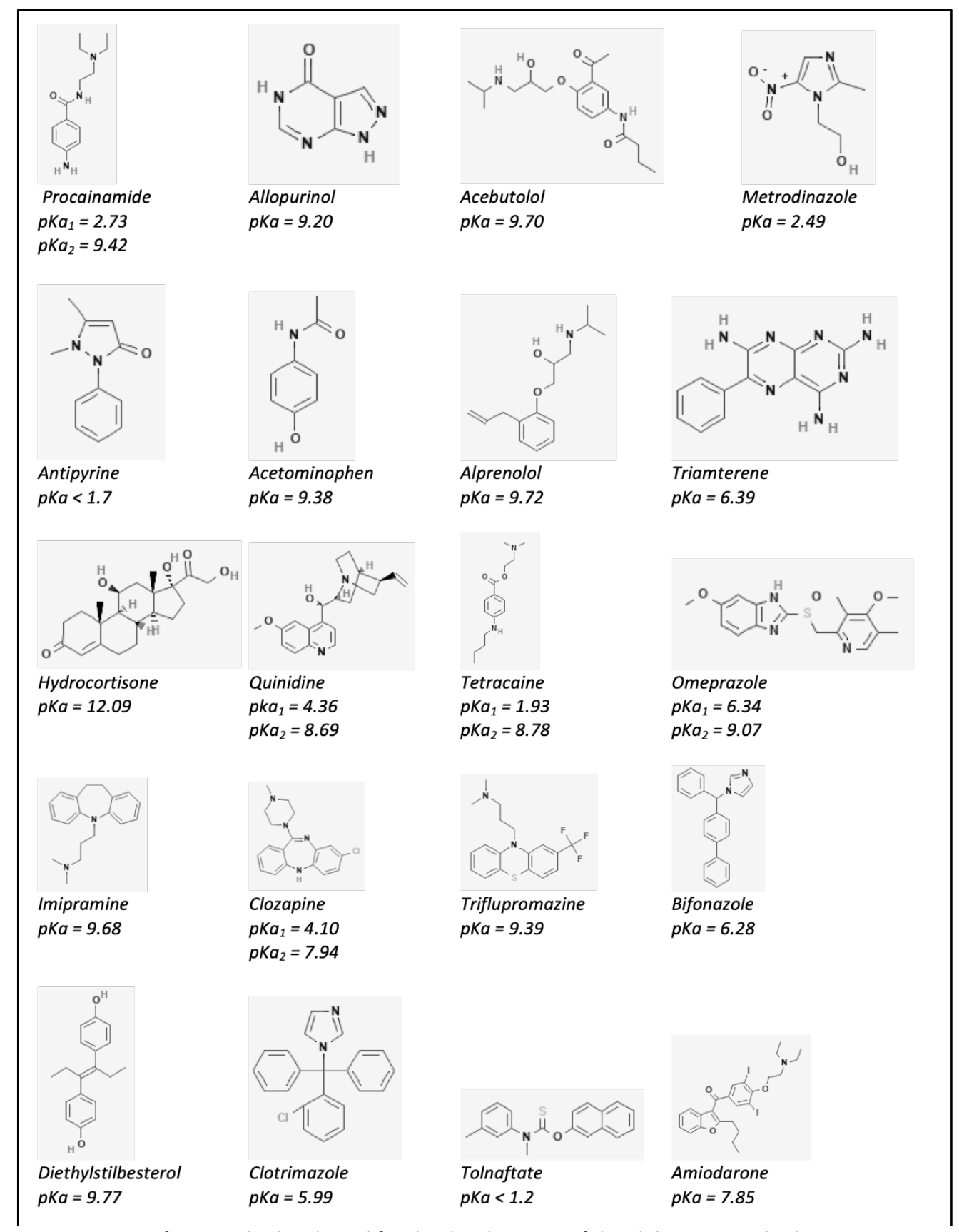

Fig. 2. Structures of 20 standards selected for the development of the alphaLogD method. 


\subsection{Correlation model between partition and retention}

Each solution of standard, initially dissolved in DMSO, is diluted down to $1 \mathrm{mM}$ in either $50 / 50 \mathrm{v} / \mathrm{v}$ or $25 / 75 \mathrm{v} / \mathrm{v}$ water/methanol mixture. The DMSO present in the injected solution is used as the void volume marker and its corresponding retention time $\left(\mathrm{t}_{0}\right)$ is included in the calculation of logk' (equation 3 ). The tetracaine is injected and eluted in each isocratic method from $20 \%$ to $75 \%$ of methyl alcohol. Based on their known lipophilicity, the standards of lipophilicity below the tetracaine lipophilicity of 2.29 are injected on the low ranges of methanol from $20 \%$ to $45 \%$, and those compounds with lipophilicity above 2.29 are injected on the high ranges of methanol from $60 \%$ to $75 \%$. Each solution is injected 3 times, and three different lots of Ascentis ${ }^{\circledR}$ Express Amide stationary phase are being tested (Figure 3 ). All the chromatographic conditions are similar to the ones applied for the study of tetracaine. The $\operatorname{logk}_{\mathrm{w}}$ of each compound is calculated with equation (4) from a curve built with at least three different solvents strengths.

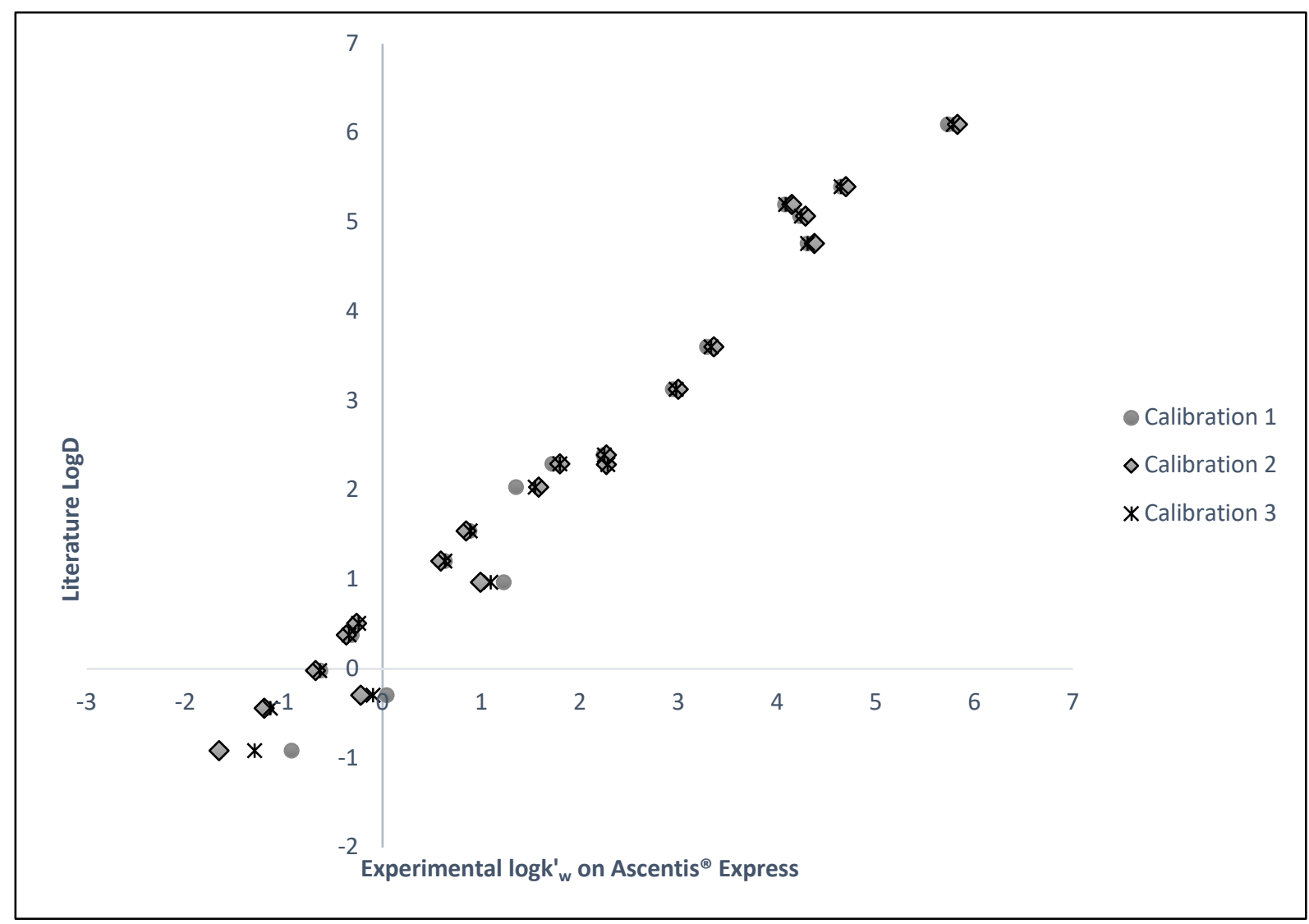

Fig. 3. Repeatability and reproducibility studies of alphaLogD calibration curves

Each linear regression and analysis of variance (ANOVA) statistics are reported in Table 3. The equality of the three linear regressions is shown as pair-wise comparisons tests for parallel lines, which includes tests for equality of slopes and intercepts, reported in Table 4. The slopes and y intercepts of the three curves are not significantly different, so they can be pooled to build one average calibration curve with the LogD in octanol as a direct function of the retention of each studied standard on the Ascentis ${ }^{\circledR}$ Express stationary phase (5): 
Table 3

Linear regression of the 3 calibration curves.

\begin{tabular}{cccc}
\hline & Calibration 1 & Calibration 2 & Calibration 3 \\
\hline Regression & $\mathrm{y}=1.0279 \mathrm{x}+0.3989$ & $\mathrm{y}=0.9804 \mathrm{x}+0.515$ & $\mathrm{y}=1.0105 \mathrm{x}+0.4375$ \\
$\mathrm{df}$ & 20 & 20 & 20 \\
$\mathrm{R}^{2}$ & 0.970 & 0.976 & 0.976 \\
Standard Error of Estimate & 0.388 & 0.329 & 0.345 \\
Analysis of Variance & $\mathrm{F}=573.282$ & $\mathrm{~F}=802.633$ & $\mathrm{~F}=729.403$ \\
& $\mathrm{P}<0.001$ & $\mathrm{P}<0.001$ & $\mathrm{P}<0.001$ \\
\hline
\end{tabular}

Power of performed test with alpha $=0.050$

Table 4

Pair-wise comparison tests for equality of slopes and Intercepts.

\begin{tabular}{cccc}
\hline & Between Curve 1 and Curve 2 & Between Curve 2 and Curve 3 & Between Curve 3 and Curve 1 \\
\hline $\begin{array}{c}\text { Test for Equality of } \\
\text { Slopes }\end{array}$ & $\mathrm{F}=0.7490$ & $\mathrm{~F}=0.3495$ & $\mathrm{~F}=0.0938$ \\
& $\mathrm{P}=0.3925$ & $\mathrm{P}=0.5581$ & \\
& & $\mathrm{P}=0.7612$ \\
$\begin{array}{c}\text { Test for Equality of } \\
\text { Intercepts }\end{array}$ & $\mathrm{F}=0.0728$ & $\mathrm{~F}=0.0489$ & $\mathrm{P}=0.0039$ \\
\hline
\end{tabular}

$\log D_{\text {oct7.4 }}=1.009( \pm 0.022) \log k_{w}^{\prime}$ Ascentis $+0.435( \pm 0.06)$

\subsection{Discussion on the alphaLogD method}

\subsubsection{Interpretation of interactions on the Ascentis ${ }^{\circledR}$ Express phase}

The slope of the equation (5) highlights differences of energies and forces, between the distribution (HPLC) and the partition (Shake-Flask) systems. The slope value close to one implies similarity of these energies between the two systems and indicates a good correlation between the octanol-water partitioning system and the chromatographic interactions of the solute with the mobile phase and with the RP-amide stationary phase. The intercept highlights the presence of secondary interactions in the chromatographic system, despite the embedded amide function and the presence of octanol that is supposed to reduce the hydrogen bond interactions of the residual silanols of the stationary phase [27]. One could argue that the presence of decylamine (used on ELogD system) would reduce these interactions, as the intercept on the ElogD calibration is slightly lower than the one on the Ascentis $®$ Express RP-Amide ( 0.21 for ABZ-Discovery and 0.45 for Ascentis $®$ Express), but the influence of these secondary interactions on the final lipophilicity values obtained on alphaLogD is not significant enough to justify the use of a reagent that is significantly detrimental to the robustness of the whole HPLC system due to re-crystallization of the decylamine in the aqueous phase over time.

The chromatographic distribution process of the solute between the mobile phase and the stationary phase seems to be enhanced by two main types of interactions. In the low lipophilicity range, the retention is mainly led by the hydrogen bonding interactions between the solutes that have hydrogen bond donors and the amide function of the stationary phase that is hydrogen bond acceptor with its carbonyl group. It has been described that polar embedded stationary phase can enhance the retention 
of polar compounds in Reversed phase HPLC even with a high ratio of aqueous phase [28] promoting high retention of phenols, amines and to a lesser extent alcohols [26].

In the high lipophilicity range, the hydrophobic interactions represent an additional contribution to the solute retention and explain why RP-amide phase is considered more retentive than a regular $\mathrm{C}_{18}$ support [26].

\subsubsection{Positive effect of Fused-Core particle}

The Ascentis ${ }^{\circledR}$ Express support is made of purified 2.7- $\mu \mathrm{m}$ superficially porous silica particles that are constituted of 1.7- $\mu \mathrm{m}$ solid silica cores and $0.5-\mu \mathrm{m}$ thick shells of $9 \mathrm{~nm}$ pores and that have been developed to allow highly efficient and fast separations, supporting high flow-rate while generating low back pressure [29]. The structure of the superficially porous particles induces a reduction of the longitudinal diffusion by 20 to $30 \%$, as now $20 \%$ of the column volume is occupied by non-porous silica thereby preventing the solute from axial diffusion. In addition, the thin layer of porous particles reduces eddy dispersion inducing a quick mass transfer of the solute in the chromatographic system leading to shorter retention times, sharper peaks and higher column efficiency compared to classic regular porous silica [21]. Fused core particles enhance the linearity of the LSS model over the range of isocratic methods in the high range of polar organic content, as the low back pressure reduces the electric field that is usually created by the alignment of mobile phase dipoles at high pressure and that is responsible for the increase in retention times [30].

\subsubsection{Ammonium acetate as ion-pairing agent}

Ion-pairing chromatography is a very powerful technique to separate entities based on their ionized forms, as the ion-pairing agent creates a layer over the hydrophobic surface to add a second dimension to the retention of the solute by creating a complex that is simultaneously dissociated in the aqueous phase. The lipophilicity measurement on the ABZ-Discovery is completed in the presence of Morpholino Propane Sulfonic acid (MOPS) for positively charged entities. The Ascentis $₫$ Express chromatographic system contains the ammonium acetate, the counter-ion being the acetate with less hydrophobic interactions than the MOPS counter-ion.

A comparison of $\log \mathrm{k}_{\mathrm{w}}$ w of the same compounds on both $A B Z$ Discovery and Ascentis $₫$ Express RP-Amide stationary phases show a good correlation between the two systems (equation 6):

$\log ^{\prime}{ }_{w}($ ABZ-Discovery $)=0.9257 \log ^{\prime}{ }_{w}($ Ascentis $®$ Express $)+0.296$

There is a similar retention of positive entities in presence of ammonium acetate on the fused-core support compared to the presence of MOPS on the porous ABZ-Discovery support, despite the different hydrophobicity between these 2 entities. It can be explained by the high rate of exchanges on the fused core support between the ion-pair that is formed with the positive form of the solutes and the acetate counter-ion and the dissociated forms in the mobile phase. The low hydrophobicity of the acetate counter-ion does not hide as much as the MOPS the embedded amide function of the support. It therefore enhances the retention of entities that have a significant number of hydrogen donors, such as the positively charge entities of the acebutolol and alprenolol that have respectively 3 and 2 hydrogen donors in their ionized state (Figure 4). 


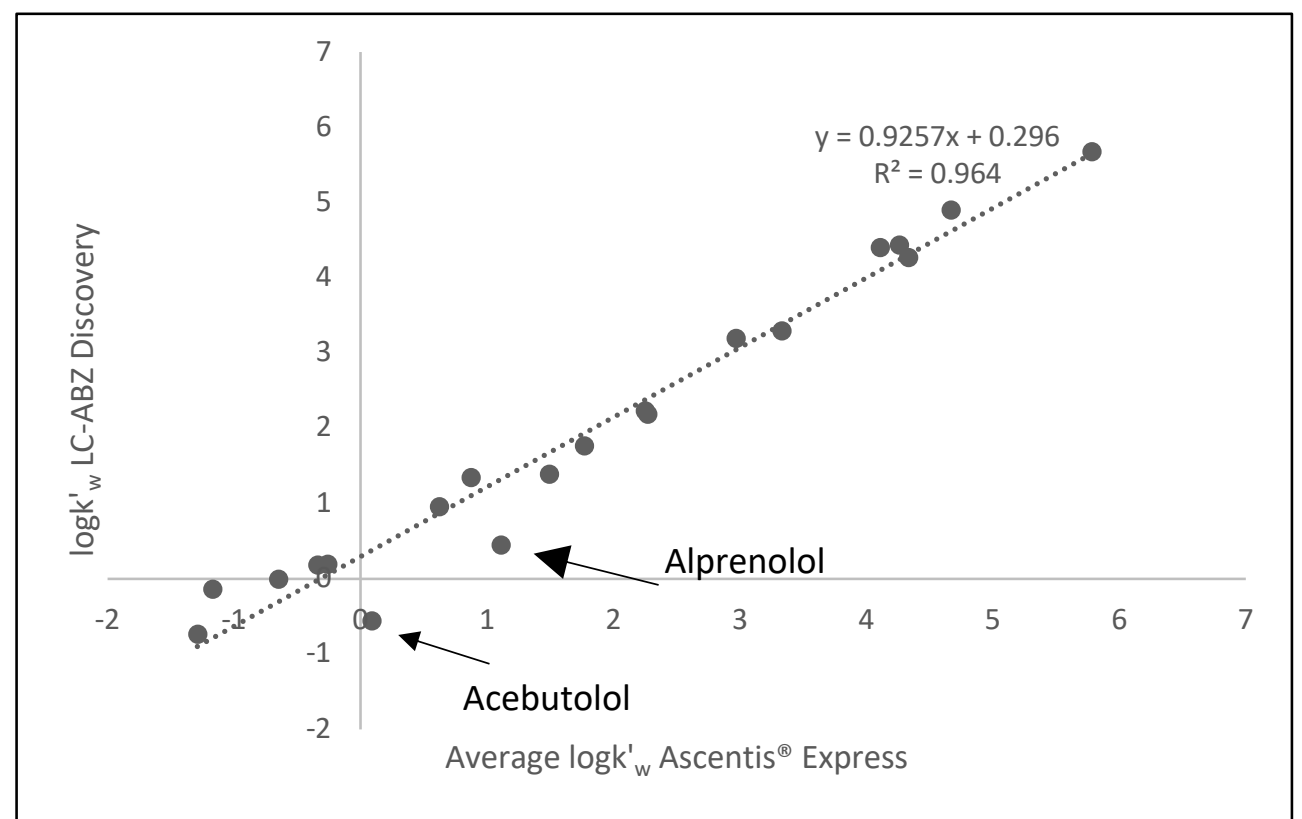

Fig. 4. Correlation of $\log ^{\prime}{ }_{w}$ Ascentis Express with $\operatorname{logk}_{w}{ }_{w}$ LC-ABZ-Discovery on standard compounds

It is important to highlight the use of ammonium acetate as both ion-pairing agent and buffer to control the $\mathrm{pH}$. It significantly simplifies the composition of the mobile phase and ensures a higher stability of the chromatographic system, a longer shelf-life of the column and the option of coupling a mass spectrometer detector for added value to the lipophilicity determination.

\subsubsection{Evaluation of AlphaLogD measurement against literature values}

A further evaluation of the alphaLogD method against the Shake-Flask method is run on the residuals between alphaLogD and Literature LogD values with the Bland Altman analysis. The Normality test of Shapiro-Wilk shows a normal distribution of differences between alphaLogD and literature LogD values. The Bland Altman Analysis (Figure 5) indicates that the alphaLogD values are on average 0.0045 lower than the literature values. In addition, the study of agreement limits leads to the conclusion that $95 \%$ of the alphaLogD measurements fall between +0.6989 and -0.7079 of the literature values.

These results show that the alphaLogD method is comparable to the Shake-Flask method. The range of alphaLogD might appear wide when compared to the Shake-Flask. It is important to remember that the Shake-Flask method is highly dependent on compound solubility in aqueous and organic phases, and that could induce significant variability in the extreme ranges of lipophilicity. 


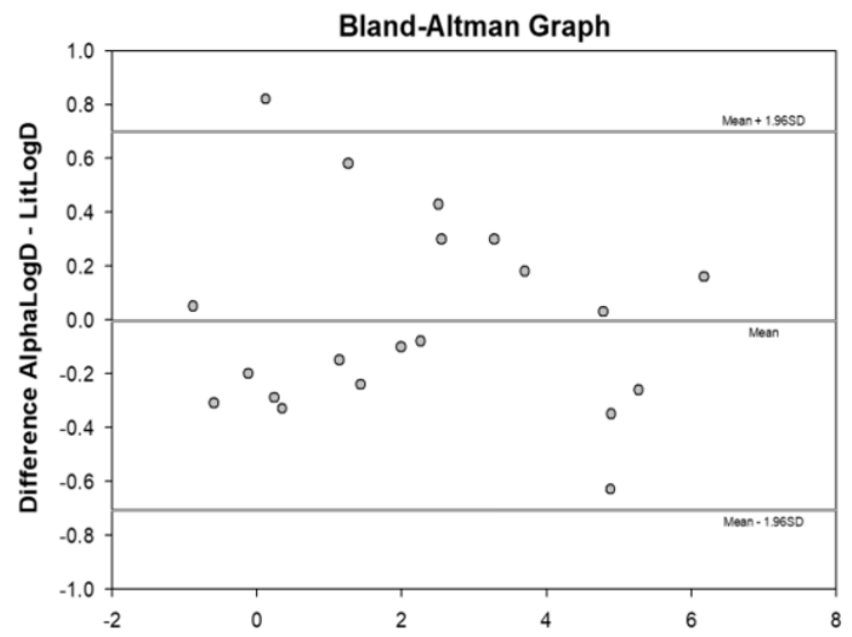

Fig. 5. Plot of the differences between alphaLogD method and Literature LogD Bias $=-0.0045$

Standard deviation $=0.3589$

Limits of agreement: lower limit LoA $=-0.7079$, upper limit UoA $=0.6989$

95\% Confidence Interval for the bias: from -0.1731 to 0.1641

95\% Confidence Interval for LoA: from -1.0000 to -0.4159

95\% Confidence Interval for UaA: from 0.4069 to 0.9910

Normality test:

alphalogD: $\quad W$-statistic $=0.957-P=0.492-$ Passed

Lit $_{\text {LogD: }} \quad$ W-statistic $=0.942-\mathrm{P}=0.258$ - Passed

Residual: $\quad$ W-statistic $=0.958-\mathrm{P}=0.514$ - Passed

\subsection{Lipophilicity measurement of Beyond Rule of 5 compounds}

Fused-core particles have been initially developed to ensure the separation of large entities due to the significant improvement of the mass transfer, allowing quick exchange between molecules and stationary phase. The chromatographic system developed with Ascentis $₫$ Express is then tested on the so called Beyond Rule of 5 molecules that are selected based on calculated properties that do not comply with the Lipinski Rule of 5 (Table 5), with at least 2 out-of-compliance rules out of 5 [31]. The applied chromatographic conditions are similar to the ones used for the small molecules with the use of isocratic methods in the high range of methanol due to the high predicted lipophilicity.

The difficulty of this specific study does not reside in the choice of the Beyond Rule of 5 standards nor in the measurement of the lipophilicity by chromatography but in finding in the literature the lipophilicity data to correlate to the experimental $\log \mathrm{k}_{\mathrm{w}}$. With predicted high lipophilicity and resulting low solubility, most of these Beyond Rule of 5 are not measurable by the shake-flask method. The calculated values don't always integrate the 3D as for the macrocycles (Figure 6), and the values of reference we use for this study are chromatographic data measured on the ELogD system [32]. 
Table 5

Calculated and measured properties of "Beyond rule of 5" molecules

\begin{tabular}{cccccccccc}
\hline & $\begin{array}{c}\text { MW } \\
\text { (g/mol) }\end{array}$ & $\begin{array}{c}\text { Rotatable } \\
\text { Bounds }\end{array}$ & $\begin{array}{c}\text { TPSA } \\
(\AA)\end{array}$ & $\begin{array}{c}\# \\
\text { Hydrogen } \\
\text { donors }\end{array}$ & $\begin{array}{c}\text { Out of } \\
\text { compliance } \\
\text { Ro5 }\end{array}$ & $\begin{array}{c}\text { Measured } \\
\text { pKa }\end{array}$ & $\begin{array}{c}\text { Calc } \\
\text { LogP } \\
\text { (ACD) }\end{array}$ & $\begin{array}{c}\text { ELogP } \\
\text { [32] }\end{array}$ & $\begin{array}{c}\text { alphaLogP** } \\
\text { Telaprevir }\end{array}$ \\
\hline 679.8 & 14 & 180 & 4 & 2 & 11.84 & 3.93 & 4.4 & 4.48 \\
Atazanavir & 704.9 & 18 & 171 & 5 & 4 & 4.29 & 5.20 & 4.7 & 4.81 \\
Ritonavir & 720.9 & 18 & 202 & 4 & 3 & 1.92 & 5.28 & 4.9 & 5.09 \\
Tacrolimus & 804 & 7 & 178 & 3 & 2 & $3.30 *$ & 3.96 & 6.1 & N/A \\
Everolimus & 958.2 & 9 & 205 & 3 & 2 & $10.40^{*}$ & 3.35 & 6.7 & 6.8 \\
Temsirolimus & 1030 & 7 & 242 & 4 & 2 & 9.96 & 2.96 & 6.9 & 7.00 \\
Zotarolimus & 966.2 & 7 & 219 & 2 & 2 & 9.81 & 3.55 & N/A & 6.59 \\
Ledipasvir & 889 & 12 & 175 & 4 & 3 & 4.32 & 6.77 & N/A & 6.99 \\
\hline
\end{tabular}

*ACD calculated pKa

**Calculated with global calibration curve

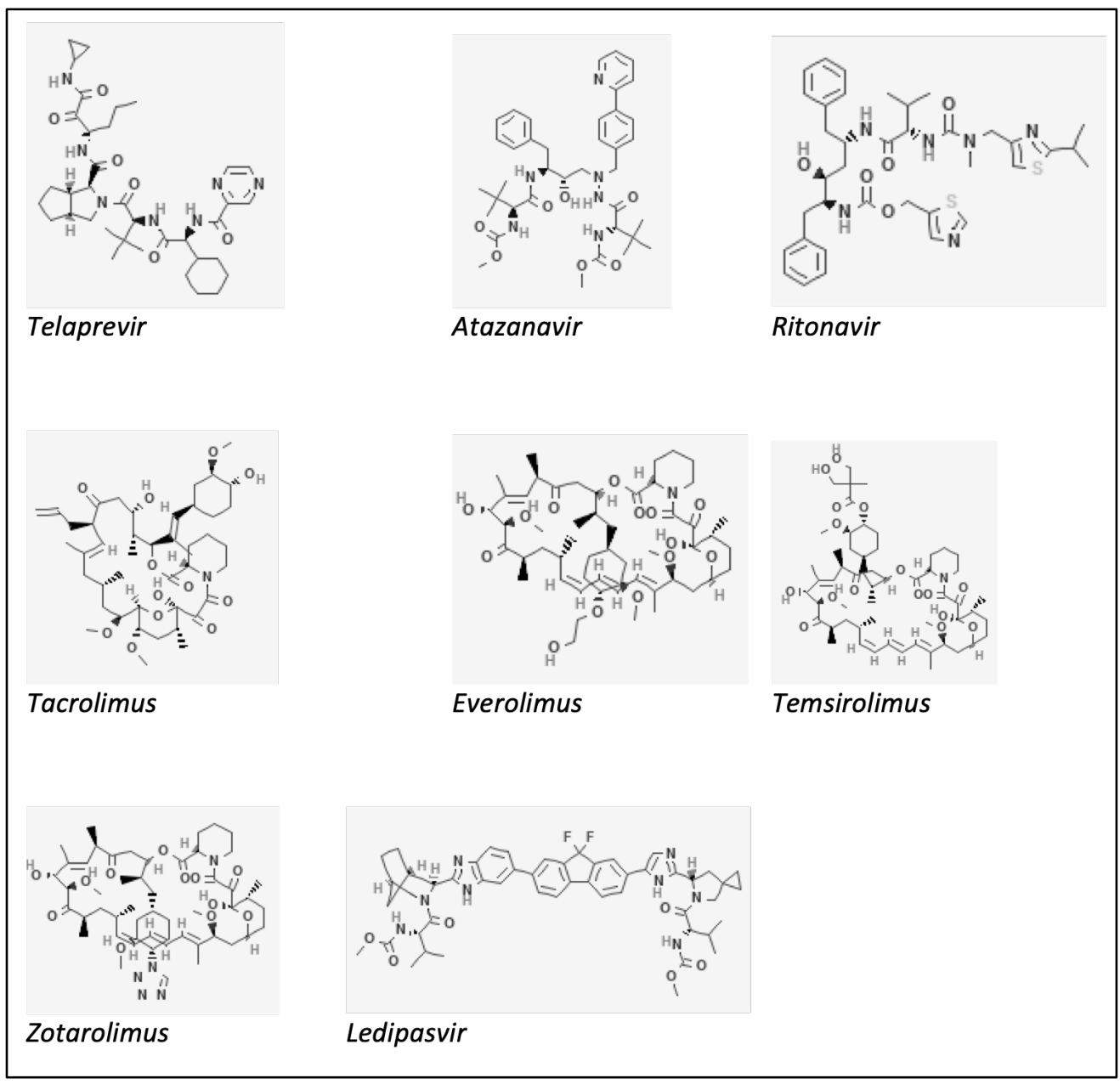

Fig. 6. Structure of "Beyond Rule of 5" molecules used as calibration standards 
The correlation of $\operatorname{logk}^{\prime}{ }_{\mathrm{w}}$ (Ascentis $®$ Express) with ELogP (as all the species are neutral at $\mathrm{pH} 7.4$ ) on the compounds presents excellent similarities of energies of interactions between the two systems as shown in Figure 7 and, as a result, we can build a calibration curve including small and large molecules (Figure 8).

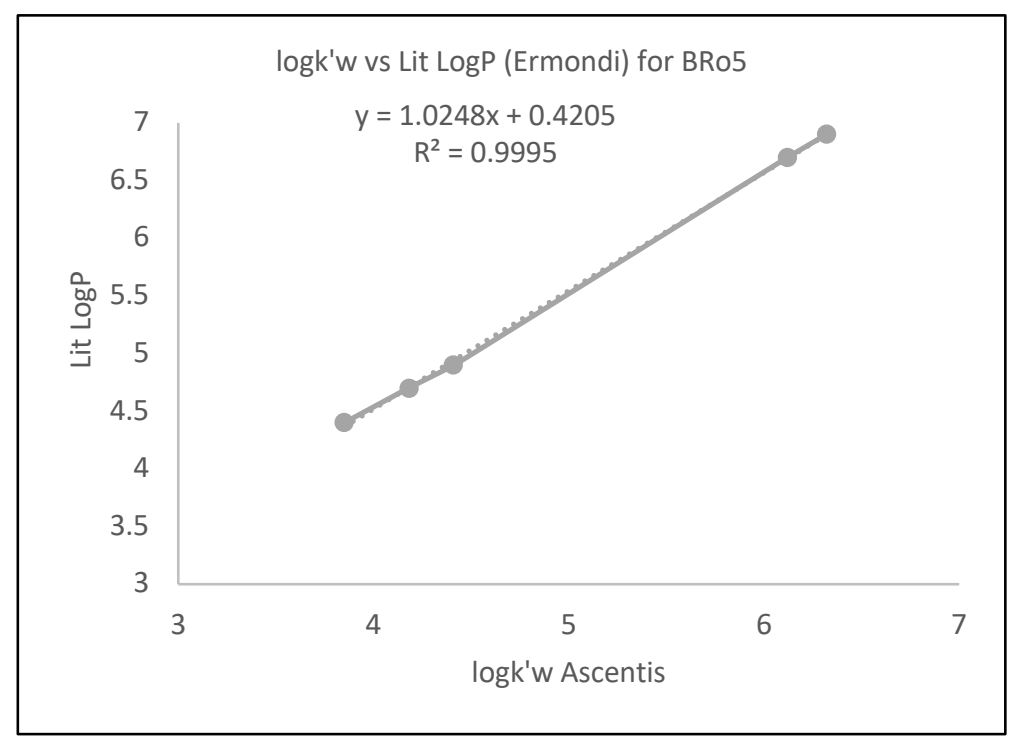

Fig. 7. Correlation $\log k^{\prime}{ }_{w}$ Ascentis $®$ Express with literature ELogP [32]

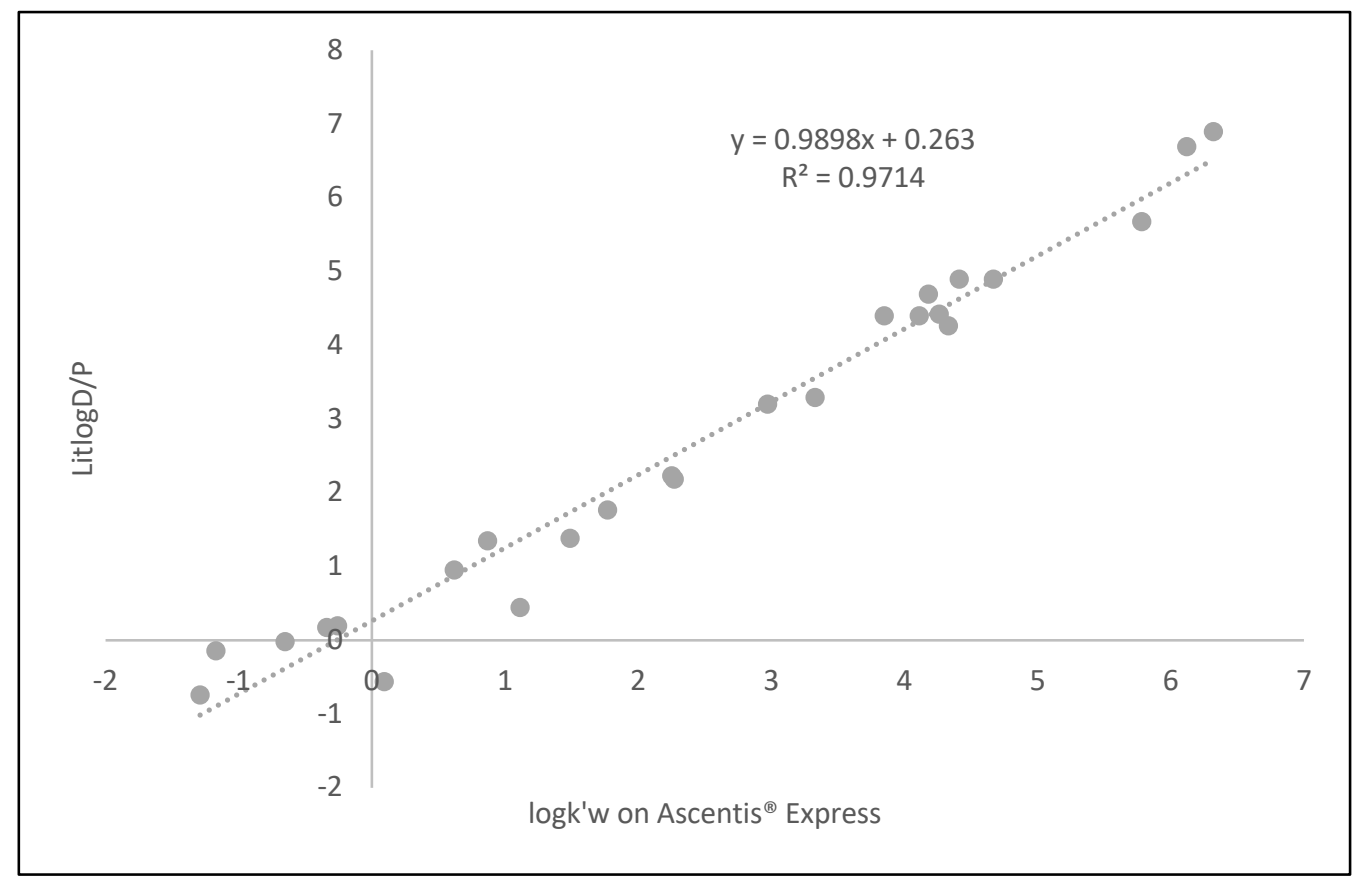

Fig. 8. Calibration curve for alphaLogD determination including small and large molecules

\subsection{Application on research compounds}

The final alphalogD method is being tested on a pool of 266 research compounds of unknown structures 
and ionization stages and is compared on the ElogD method (Figure 9).

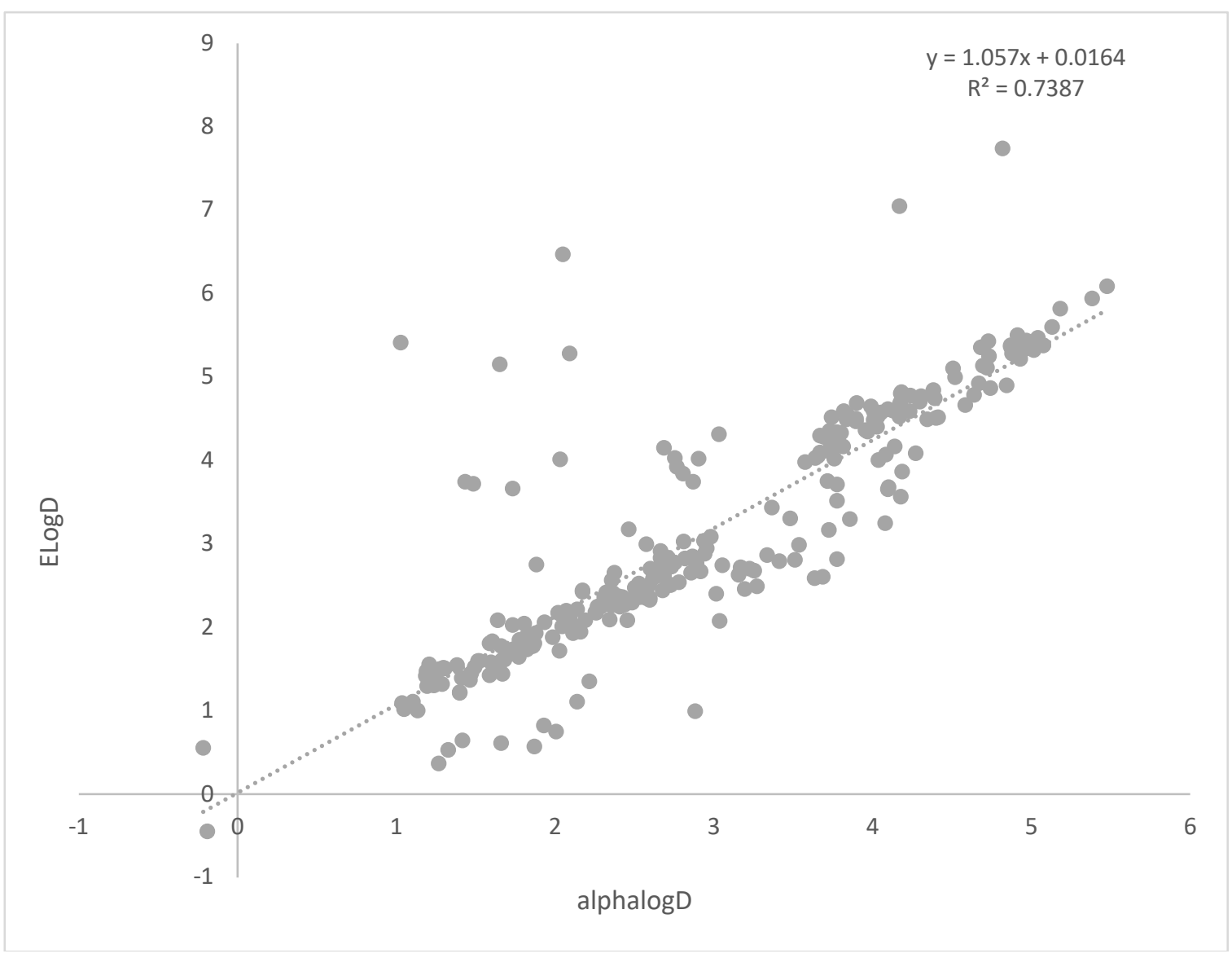

Fig. 9. Research compounds lipophilicity measurement with alphaLogD versus ELogD

The analysis of alphaLogD data compared to the ELogD data shows a good correlation between the two methods in the low lipophilicity range as well as in the high range. The outliers in the low range might be due to stability issues of the compounds as there was a lag time between the analysis with the two methods. The main difference between the data resides in the lipophilicity range from 3 to 4 . The observed granularity of the alphaLogD data in this range could be interpreted, in the absence of structural information, by the higher selectivity of the SPP support regarding the hydrophobic interactions that are translated with different retention times compared to the LC-ABZ-Discovery support.

\section{Conclusion}

The alphaLogD HPLC method has been developed on the Superficially Porous Particle to simplify the existing ELogD method with a less complex mobile phase and an improved shelf-life of the column with similar results for the lipophilicity range from 0 to 3 . The fused-core particles with small particle size leverage the rate of exchanges between the solutes and the stationary phase. The $\mathrm{C}_{16}$-amide chemistry of the support enhances hydrogen bond interactions with the analytes, in addition to hydrophobic 
interactions, to mimic the octanol-water partition. The overall combination of increased equilibriums and selective interactions leads to the use of a lower number of isocratic methods to reliably determine the logk' ${ }_{w}$ of compounds. The power of the HPLC method increases the analysis throughput by $40 \%$ and expands the lipophilicity range up to 7 to confidently characterize Beyond-Rule-of- 5 molecules. Finally, the use of ammonium acetate in the mobile phase, compared to the phosphate buffer in the ELogD method, allows the hyphenation to mass spectrometry detection to determine the lipophilicity with higher accuracy.

\section{Acknowledgments}

We thank Aimee Kestranek and Kate Favre for their constant support and for allocating time to the team to run the method development and optimization.

We thank Wendy Roe and Cory Muraco for Millipore Sigma for giving us access to a free Superficially Porous Particle Ascentis ${ }^{\circledR}$ Express column to allow us starting the alphaLogD method development and optimization.

\section{References}

[1] X. Liu, B. Testa, A. Fahr, Lipophilicity and its relationship with passive drug permeation, Pharm. Res. 28 (2011) 962-977. https://doi.org/10.1007/s11095-010-0303-7.

[2] M.J. Waring, Lipophilicity in drug discovery, Expert Opin. Drug Discov. 5 (2010) 235248. https://doi.org/10.1517/17460441003605098.

[3] M.V.S. Varma, B. Feng, R.S. Obach, M.D. Troutman, J. Chupka, H.R. Miller, A. ElKattan, Physicochemical determinants of human renal clearance, J. Med. Chem. 52 (2009) 4844-4852. https://doi.org/10.1021/jm900403j.

[4] T.W. Johnson, K.R. Dress, M. Edwards, Using the Golden Triangle to optimize clearance and oral absorption, Bioorganic Med. Chem. Lett. 19 (2009) 5560-5564.

https://doi.org/10.1016/j.bmcl.2009.08.045.

[5] Y. Henchoz, B. Bard, D. Guillarme, P.A. Carrupt, J.L. Veuthey, S. Martel, Analytical tools for the physicochemical profiling of drug candidates to predict absorption/distribution, Anal. Bioanal. Chem. 394 (2009) 707-729. https://doi.org/10.1007/s00216-009-2634-y.

[6] V.H. Thomas, S. Bhattachar, L. Hitchingham, P. Zocharski, M. Naath, N. Surendran, C.L. Stoner, A. El-Kattan, The road map to oral bioavailability: an industrial perspective, Expert Opin. Drug Metab. Toxicol. 2 (2006) 591-608. https://doi.org/10.1517/17425255.2.4.591.

[7] M.J. Waring, C. Johnstone, A quantitative assessment of hERG liability as a function of lipophilicity, Bioorg. Med. Chem. Lett. 17 (2007) 1759-1764. https://doi.org/https://doi.org/10.1016/j.bmcl.2006.12.061.

[8] D.A. Price, J. Blagg, L. Jones, N. Greene, T. Wager, Physicochemical drug properties associated with in vivo toxicological outcomes: a review, Expert Opin. Drug Metab. Toxicol. 5 (2009) 921-931. https://doi.org/10.1517/17425250903042318.

[9] C.A. Lipinski, F. Lombardo, B.W. Dominy, P.J. Feeney, Experimental and computational approaches to estimate solubility and permeability in drug discovery and development settings1PII of original article: S0169-409X(96)00423-1. The article was originally published in Advanced Drug Delivery Reviews 23 (1997) 3, Adv. Drug Deliv. Rev. 46 (2001) 3-26. https://doi.org/https://doi.org/10.1016/S0169-409X(00)00129-0. 
[10] T. Rezai, J.E. Bock, M. V Zhou, C. Kalyanaraman, R.S. Lokey, M.P. Jacobson, Conformational Flexibility, Internal Hydrogen Bonding, and Passive Membrane Permeability: Successful in Silico Prediction of the Relative Permeabilities of Cyclic Peptides, J. Am. Chem. Soc. 128 (2006) 14073-14080. https://doi.org/10.1021/ja063076p.

[11] G. Caron, M. Vallaro, G. Ermondi, Log P as a tool in intramolecular hydrogen bond considerations, Drug Discov. Today Technol. 27 (2018) 65-70. https://doi.org/https://doi.org/10.1016/j.ddtec.2018.03.001.

[12] K.D. Freeman-Cook, R.L. Hoffman, T.W. Johnson, Lipophilic efficiency: the most important efficiency metric in medicinal chemistry, Future Med. Chem. 5 (2013) 113115. https://doi.org/10.4155/fmc.12.208.

[13] T.W. Johnson, R.A. Gallego, M.P. Edwards, Lipophilic Efficiency as an Important Metric in Drug Design, J. Med. Chem. 61 (2018) 6401-6420. https://doi.org/10.1021/acs.jmedchem.8b00077.

[14] M.D. Trone, M.S. Leonard, M.G. Khaledi, Congeneric Behavior in Estimations of Octanol-Water Partition Coefficients by Micellar Electrokinetic Chromatography, Anal. Chem. 72 (2000) 1228-1235. https://doi.org/10.1021/ac9908521.

[15] C. Giaginis, A. Tsantili-Kakoulidou, Current State of the Art in HPLC Methodology for Lipophilicity Assessment of Basic Drugs. A Review, J. Liq. Chromatogr. I\& Relat. Technol. 31 (2007) 79-96. https://doi.org/10.1080/10826070701665626.

[16] B. Zheng, L.M. West, ESTIMATING THE LIPOPHILICITY OF NATURAL PRODUCTS USING A POLYMERIC REVERSED PHASE HPLC METHOD, J. Liq. Chromatogr. I\& Relat. Technol. 33 (2009) 118-132. https://doi.org/10.1080/10826070903430464.

[17] L. Grumetto, C. Carpentiero, P. Di Vaio, F. Frecentese, F. Barbato, Lipophilic and polar interaction forces between acidic drugs and membrane phospholipids encoded in IAMHPLC indexes: Their role in membrane partition and relationships with BBB permeation data, J. Pharm. Biomed. Anal. 75 (2013) 165-172. https://doi.org/https://doi.org/10.1016/j.jpba.2012.11.034.

[18] K. Valko, Biomimetic chromatography to accelerate drug discovery: Part I, LC-GC North Am. 36 (2018) 520-526.

[19] K.B.T.-L.E. Valko, Biomimetic Chromatography to Accelerate Drug Discovery; part II, $31(2018) 62+$. https://link.gale.com/apps/doc/A551543372/AONE?u=umuser\&sid=AONE\&xid=1a20fdd a.

[20] F. Lombardo, M.Y. Shalaeva, K.A. Tupper, F. Gao, ElogDoct: A Tool for Lipophilicity Determination in Drug Discovery. 2. Basic and Neutral Compounds, J. Med. Chem. 44 (2001) 2490-2497. https://doi.org/10.1021/jm0100990.

[21] S. Fekete, E. Oláh, J. Fekete, Fast liquid chromatography: The domination of core-shell and very fine particles, J. Chromatogr. A. 1228 (2012) 57-71.

https://doi.org/10.1016/j.chroma.2011.09.050.

[22] M.J. Kamlet, R.M. Doherty, M.H. Abraham, Y. Marcus, R.W. Taft, Linear solvation energy relationship. 46. An improved equation for correlation and prediction of octanol/water partition coefficients of organic nonelectrolytes (including strong hydrogen bond donor solutes), J. Phys. Chem. 92 (1988) 5244-5255. https://doi.org/10.1021/j100329a035.

[23] A. Pagliara, E. Khamis, A. Trinh, P.-A. Carrupt, R.-S. Tsai, B. Testa, Structural Properties 
Governing Retention Mechanisms on RP-HPLC Stationary Phases Used for Lipophilicity Measurements, J. Liq. Chromatogr. 18 (1995) 1721-1745. https://doi.org/10.1080/10826079508010002.

[24] N.C. Dias, M.I. Nawas, C.F. Poole, Evaluation of a reversed-phase column (Supelcosil LC-ABZ) under isocratic and gradient elution conditions for estimating octanol-water partition coefficients, Analyst. 128 (2003) 427-433. https://doi.org/10.1039/B300574G.

[25] D. Benhaim, E. Grushka, Characterization of Ascentis RP-Amide column: Lipophilicity measurement and linear solvation energy relationships, J. Chromatogr. A. 1217 (2010) 65-74. https://doi.org/https://doi.org/10.1016/j.chroma.2009.11.013.

[26] D. Bell, Alternative Selectivity of Amide-based Embedded Polar Group HPLC Stationary Phases - Role of Hydrogen Bonding Linear Solvation Energy Relationship ( LSER ) Study Separation of Catechols and Resorcinols, (2021) 1-5.

[27] C. Giaginis, S. Theocharis, A. Tsantili-Kakoulidou, Octanol/water partitioning simulation by reversed-phase high performance liquid chromatography for structurally diverse acidic drugs: Effect of n-octanol as mobile phase additive, J. Chromatogr. A. 1166 (2007) 116125. https://doi.org/https://doi.org/10.1016/j.chroma.2007.08.004.

[28] Y. Guo, S. Gaiki, Retention behavior of small polar compounds on polar stationary phases in hydrophilic interaction chromatography, J. Chromatogr. A. 1074 (2005) 71-80. https://doi.org/https://doi.org/10.1016/j.chroma.2005.03.058.

[29] J.J. DeStefano, T.J. Langlois, J.J. Kirkland, Characteristics of Superficially-Porous Silica Particles for Fast HPLC: Some Performance Comparisons with Sub-2- $\mu$ m Particles, J. Chromatogr. Sci. 46 (2008) 254-260. https://doi.org/10.1093/chromsci/46.3.254.

[30] A. Andrade-Eiroa, Reverse-High Performance Liquid Chromatography Mechanism Explained by Polarization of Stationary Phase, CheM. 1 (2011) 62-79. https://doi.org/10.5618/chem.2011.v1.n1.8.

[31] C.A. Lipinski, Lead- and drug-like compounds: the rule-of-five revolution, Drug Discov. Today Technol. 1 (2004) 337-341. https://doi.org/https://doi.org/10.1016/j.ddtec.2004.11.007.

[32] G. Ermondi, M. Vallaro, G. Goetz, M. Shalaeva, G. Caron, Experimental lipophilicity for beyond Rule of 5 compounds, Futur. Drug Discov. 1 (2019) FDD10. https://doi.org/10.4155/fdd-2019-0002. 